\title{
A Transição Epidemiológica no Brasil
}

\section{The Epidemiologic Transition in Brazil}

\section{Pedro Reginaldo Prata ${ }^{\prime}$}

PRATA, P. R. The Epidemiologic Transition in Brazil. Cad. Saude Puibl., Rio de Janeiro, 8 (2): 168-175, abr/jun, 1992.

The author critically evaluates the evolution of the Brazilian mortality pattern during the last fifty years under the framework of the epidemiologic transition theory. The author also discusses mortality determination as a result of economic development and preventive measures. He considers that cardiovascular diseases, neoplasms and injuries are related with environmental and socio-cultural factors and therefore cannot be considered chronic degenerative diseases but preventable ones. These groups of disease are also considered to be unevenly distributed and more prevalent in deprived populations. The author also refers to the simultaneous prevalence of two distinct epidemiologic patterns described in the epidemiologic transition theory, due to the remaining infectious diseases prevalence or to the outbreak of previously eradicated infectious diseases in Brazil, leading to an incomplete epidemiologic transition.

Keywords: Epidemiologic Transition; Mortality and Morbidity Determinants; Mortality Pattern

\section{INTRODUÇÃO}

O perfil de morbi-mortalidade pode ser considerado um indicador relativamente sensivel das condições de vida e do modelo de desenvolvimento de uma população, sendo o resultado da interação de diversos fatores interdependentes. Wood \& Carvalho (1988), por exemplo, consideram que os modos de produção econômica $e$ de reprodução humana interagem para determinar a estrutura econômica e demográfica (fertilidade, mortalidade e migração) de uma população. Além disso, fatores ambientais e sócio-culturais devem ser considerados, não sendo possivel, portanto, separar o nível de mortalidade de sua estrutura e de sua relação com fatores históricos, sócio-econômicos, demográficos e ambientais.

Tal como ocorreu anteriormente na Europa, o declínio do coeficiente de mortalidade geral não é o único aspecto notável no Brasil nos últimos $\mathbf{5 0}$ anos; também a redução da mortalidade infantil, o aumento da expectativa de vida

\footnotetext{
'Departamento de Saude Pública, Universidade Federal do Mato Grosso do Sul. Campus Universitário, Campo Grande, MS, 79070-900, Brasil.
}

da população e a modificação do seu perfil epidemiológico foram observados. Este processo, o qual Omran (1971) descreveu como de transição epidemiológica, caracteriza-se pela evolução progressiva de um perfil de alta mortalidade por doenças infecciosas para um outro onde predominam os óbitos por doenças cardiovasculares, neoplasias, causas externas e outras doenças consideradas crônico-degenerativas.

\section{A MORTALIDADE E SEUS DETERMINANTES}

$\mathrm{Na}$ Europa, as Revoluçōes Agrícola e Industrial, a urbanização e a melhoria das condições de vida durante os últimos séculos foram responsáveis por um importante declínio da mortalidade e pela modificação do perfil epidemiológico da população, numa época de pouco conhecimento médico e de implementação limitada de medidas terapêuticas eficazes.

Tomando como exemplo a Inglaterra, observa-se que, no século XVIII, o principal fator responsável pelo declínio da taxa de mortalidade foi a redução das doenças infecciosas, graças 
a melhores níveis de nutrição alcançados com a Revolução Agricola e com melhorias estritamente ambientais (McKeown \& Brown, 1956).

No século XIX, também na Inglaterra, três quartos da redução da taxa de mortalidade deveram-se à menor prevalência da tuberculose, como conseqüência da melhoria das condiçōes de vida trazida pela Revolução Industrial. $O$ resto do declínio da mortalidade deveu-se à introdução de medidas de saúde pública que diminuíram a incidência do tifo, da febre tifóide e do cólera (McKeown \& Record, 1963).

No entanto, nos países desenvolvidos, neste século XX, além da continuada melhoria dos níveis nutricionais da população, três quartos da redução da taxa de mortalidade deveram-se ao controle das doenças infecciosas ainda persistentes pela introdução de medidas médicas (imunização e tratamento) e pela redução da exposição da população ao risco de infecções, graças à melhoria dos serviços de saneamento (água, esgoto e destino de resíduos) e de vigilância sanitária (manipulação adequada de alimentos) (McKeown et al., 1975).

$E$ interessante observar que o inverso ocorreu no continente africano durante este período, particularmente a partir do século XIX. Enquanto a expansão colonialista foi, em grande parte, responsável pelo aumento da oferta de alimentos, em quantidade e qualidade, na Europa, a modificação das culturas tradicionais e da estrutura produtiva por parte do colonizador reduziu a oferta de alimentos localmente. Além disso, a modificação da forma de ocupação do espaço territorial e da relação humana com o meio ambiente facilitou a ocorrência de epidemias e a prevalência de endemias (antes de pouca importância), graças ao desequilíbrio ecológico então introduzido na região (Doyal, 1979).

Atualmente, no mundo em desenvolvimento, Preston (1975) considera que o determinismo econômico da mortalidade não seria tão importante, graças à introdução de medidas efetivas de proteção à saúde. Desta forma, como consequiência de técnicas preventivas de relativo baixo custo e da melhoria do nível educacional da população, alguns países não-industrializados da Africa e da Ásia experimentaram uma redução dos niveis de mortalidade, enquanto permaneciam no contexto de sociedades agrárias pouco desenvolvidas.

Na América Latina, Arriaga \& Davis (1969) consideram que o impacto do desenvolvimento econômico no declínio da mortalidade foi importante no início do século. Entretanto, depois dos anos 30, o declínio da mortalidade foi muito mais rápido do que aquele esperado pelo nível regional de desenvolvimento. Os autores, à semelhança de Preston, concluíram que medidas de saúde pública e técnicas de medicina preventiva foram então responsáveis por este declínio.

Inversamente, Pendleton (1985), ao estudar os estágios de transição demográfica das sociedades em desenvolvimento durante o século $\mathrm{XX}$, concluiu que, no estágio inicial da transição, o declínio da mortalidade se dá, principalmente, através de medidas de saúde, e quando estas naçōes atingem uma menor taxa de mortalidade, o desenvolvimento econômico passa a ser o responsável pelo declínio subseqüente da mortalidade infantil e pelo aumento da expectativa de vida nestas sociedades.

Estas duas interpretações sobre se o declínio da mortalidade está relacionado com o desenvolvimento econômico ou com intervenções de saúde não estão necessariamente incorretas, mas incompletas, porque não são excludentes, já que a determinação do perfil epidemiológico da mortalidade deve ser considerada como o resultado de um processo dinâmico, onde as variáveis são interdependentes e podem ter um peso diferenciado, de acordo com o local, com a sociedade e com o tempo histórico. De fato, no Brasil, ambos os mecanismos de mudança do padrão de mortalidade (a adoção de medidas preventivas e o desenvolvimento econômico) ocorrem simultaneamente, como salientaram Wood \& Carvalho (1988: 88):

o passo e a velocidade da mudança na mortalidade nos últimos 40 anos sugerem que tanto a difusão de tecnologias de controle quanto a melhoria no padrāo de vida tiveram o seu papel. 


\section{A EVOLUÇÃO DO PERFIL EPIDEMIOLÓGICO DA MORTALIDADE NO BRASIL}

No Brasil, a taxa geral de mortalidade decresceu de $18 / 1000$, em 1940 , para uma taxa estimada entre $6 / 1000$ e $8 / 1000$ em 1985; a expectativa de vida cresceu 20 anos no mesmo periodo, e a mortalidade infantil decresceu de $160 / 1000$, em 1940, para $85 / 1000$, em 1980. Já o estudo da mortalidade por causas no período de 1930 a 1985 (capitais) evidencia uma similaridade ao ocorrido anteriormente na Europa. Em 1930 , as doenças infecciosas e parasitárias foram responsáveis por $46 \%$ do total de óbitos, enquanto em 1985 elas representavam apenas 7\%. Por outro lado, as doenças do apa- relho circulatório representavam $12 \%$ em 1930 e chegaram a $33 \%$ em 1985. Aumentos ainda mais pronunciados ocorreram com as neoplasias e com as mortes conseqüentes às causas externas: ambas tiveram um aumento proporcional de $3 \%$ para $12 \%$ (Prata, 1989).

As Figuras 1 a 5 comparam, nas regiōes brasileiras, a evolução da mortalidade proporcional por grupos de causas, selecionados entre os que tiveram significante variação no período de 1930 a 1985, ilustrando o processo de mudança gradativa da importância de cada grupo e as variações regionais ao longo do tempo. A evolução foi similar nas diversas regiōes, embora diferenças quantitativas sejam observadas (as figuras não totalizam $100 \%$ porque as demais causas não-selecionadas não estão ilustradas).

FIGURA 1 - Mortalidade Proporcional, Norte/1930-85

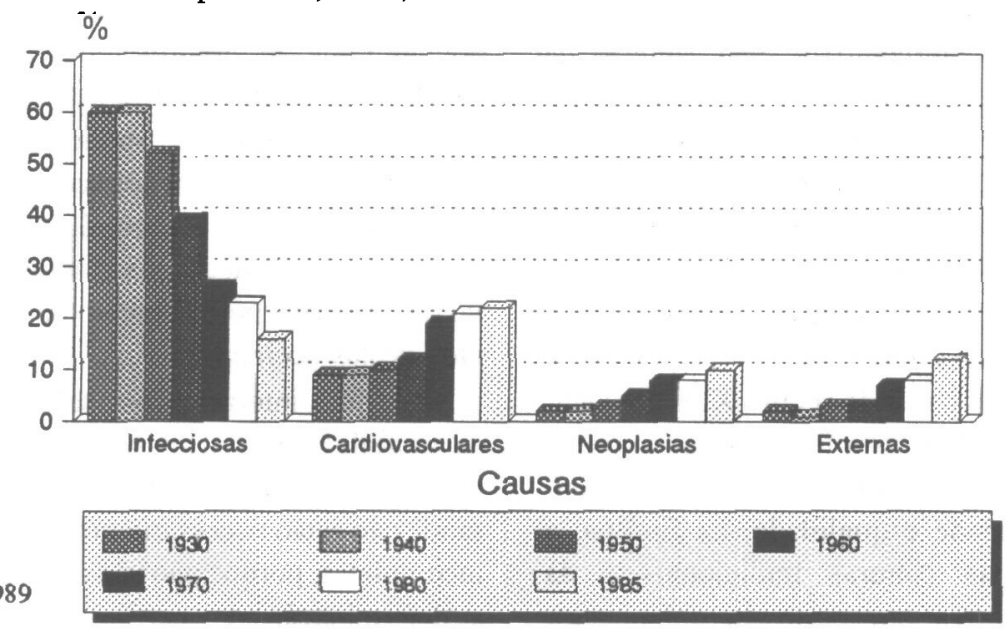

FIGURA 2 - Mortalidade Proporcional, Nordeste/1930-85

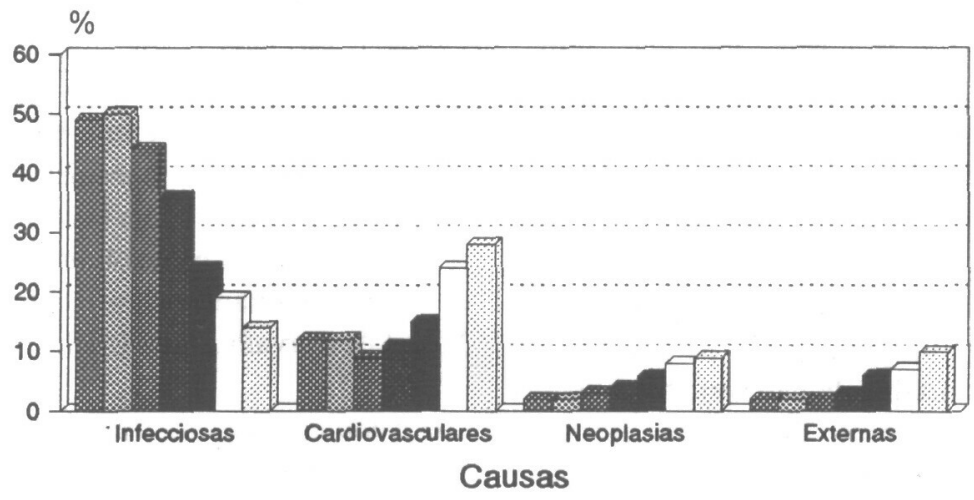

Fonte: Prata, 1989.

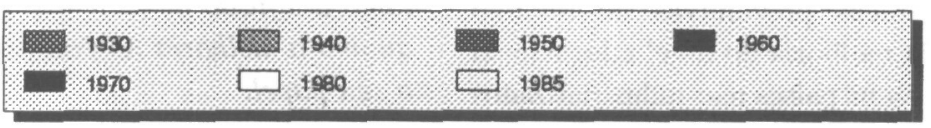


Em 1985, embora as doenças do aparelho circulatório tenham se tornado a primeira causa de morte em todas as regiões brasileiras, elas correspondiam a $37 \%$ no Sul, Centro-Oeste e Sudeste, a $28 \%$ no Nordeste, e a $22 \%$ na região Norte, enquanto as doenças infecciosas e parasitárias correspondiam a $16 \%$ na região Norte, a $14 \%$ no Nordeste, a $8 \%$ no Centro-Oeste, a apenas 5\% na região Sul, e a $4 \%$ no Sudeste. Já as neoplasias correspondiam a $17 \%$ de todas as mortes na região Sul, a $14 \%$ no Sudeste, a $11 \%$ no Centro-Oeste, a $10 \%$ na região Norte, e a 9\% no Nordeste. Enquanto isto, as causas externas correspondiam a $17 \%$ no Centro-Oeste, a $12 \%$ na região Norte e no Sudeste, a $11 \%$ no
Sul, e a $10 \%$ no Nordeste.

Em 1930, o perfil epidemiológico era bastante diferente: as doenças infecciosas eram a primeira causa de morte em todas as regiōes, correspondendo a $60 \%$ das causas na região Norte, a $49 \%$ no Nordeste, a $43 \%$ no Sudeste, a $40 \%$ no Centro-Oeste, e a $39 \%$ na região Sul. Naquela época, as doenças do aparelho circulatório eram responsáveis por apenas $13 \%$ dos óbitos no Sul, $12 \%$ no Sudeste e Nordeste, e $9 \%$ na região Norte. Já as causas externas, ao contrário do que hoje se observa, tinham pouca relevância: correspondiam a 3\% das mortes no Sudeste, a $2 \%$ no Norte, Nordeste e Sul, e a apenas $1 \%$ na região Centro-Oeste.

FIGURA 3 - Mortalidade Proporcional, Sudeste/1930-85

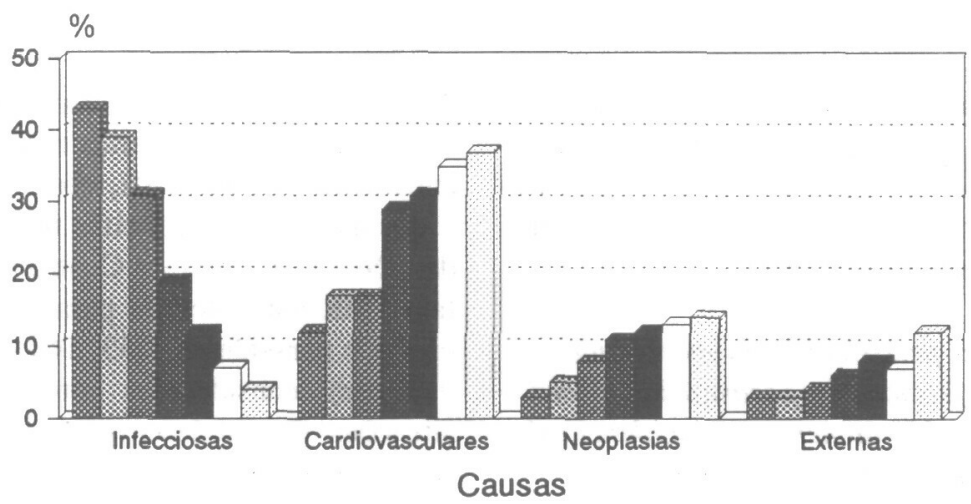

Fonte: Prata, 1989.

\begin{tabular}{|c|c|c|c|c|}
\hline & 1930 & 1940 & 1950 & 1960 \\
\hline & 1970 & $\square 1980$ & E 1985 & \\
\hline
\end{tabular}

FIGURA 4 - Mortalidade Proporcional, Sul/1930-85

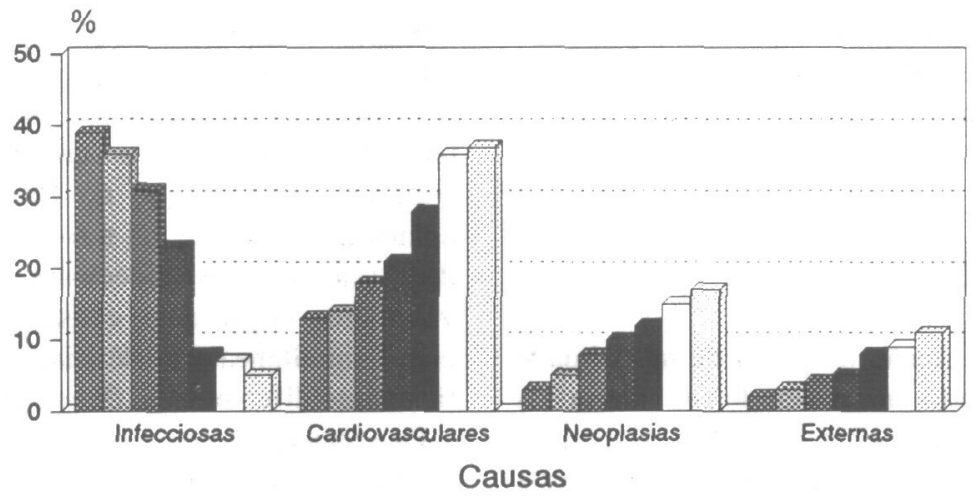

Fonte: Prata, 1989.

\begin{tabular}{|c|c|c|c|c|c|}
\hline 161930 & ck & 1940 & 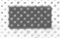 & 1950 & 1960 \\
\hline 4970 & $\square$ & 1980 & 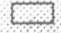 & 1985 & \\
\hline
\end{tabular}


FIGURA 5 - Mortalidade Proporcional, Centro-Oeste/1930-85
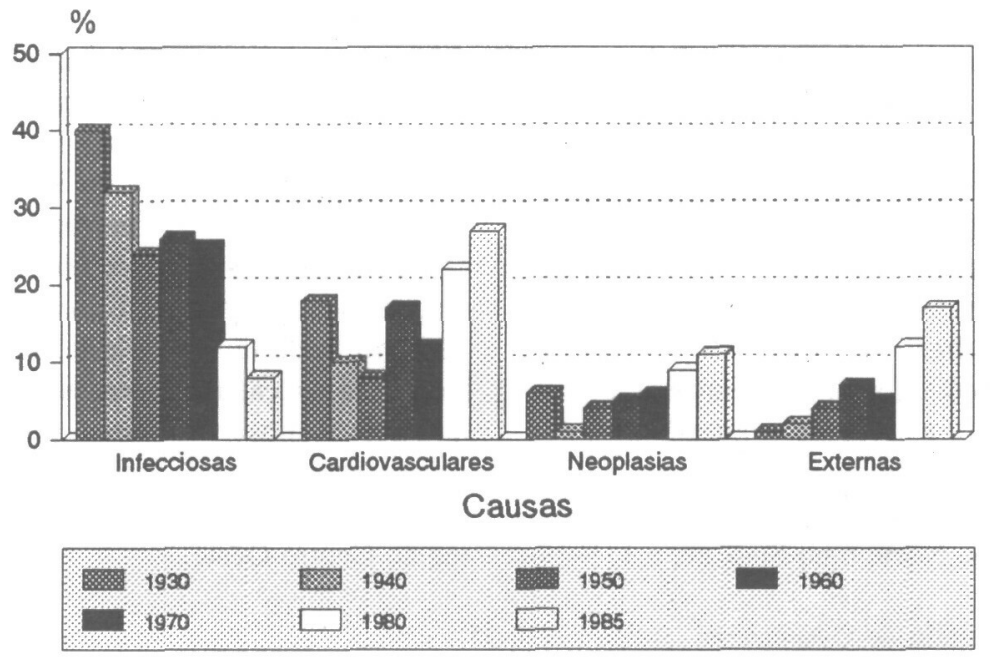

Fonte: Prata, 1989.

A semelhante conclusão chegou Possas (1989), tendo, no entanto, observado que a queda da mortalidade por doenças infecciosas não tem sido acompanhada pela redução de sua morbidade. A autora observa:

\section{Constata-se a persistência da morbidade} por doenças infecciosas e parasitárias, observando-se expressivo aumento da incidéncia da maior parte em algumas regiōes do país.

O Brasil se encontraria, portanto, em pleno estágio intermediário de transição epidemiológica, como também observou Laurenti (1990), ao concluir que esta transição nāo é uniforme:

Em alguns estados, ou regiões destes, esta se encontra em fase inicial; em outros, na fase intermediária, e em alguns a transição está quase se completando.

\section{CONCLUSÕES}

Apesar da evolução do perfil de mortalidade estar obedecendo ao esperado na teoria da transição epidemiológica em todas as regiōes brasileiras, a desigualdade processual desta evoluçāo pode ser observada, por exemplo, através do cálculo da Standard Mortality Ratio - SMR (Método Indireto de Padronização), por causas, nas diversas regiões brasileiras.
Tendo como padrão a razāo 1 (SMR = 100), onde não haveria diferença entre a mortalidade encontrada e a esperada, observa-se na Tabela 1 que as regiões brasileiras menos desenvolvidas (Norte, Nordeste e Centro-Oeste) apresentam SMR mais elevada para as doenças infecciosas e parasitárias $(253,168$ e 159, respectivamente), consideradas causas de morte em situações de subdesenvolvimento, pobreza e privação, e mais baixas para as doenças do aparelho circulatório $(25,56$ e 89 , respectivamente) e para as neoplasias $(75,51$ e 87 , respectivamente). Estas diferenças foram consideradas estatisticamente significantes para um intervalo de confiança de 95\% (Prata, 1989).

É de se esperar que este mesmo contraste seja observado entre áreas de desenvolvimento diferenciado intra-regionais e entre subgrupos populacionais submetidos a condições de vida também diferenciadas nestas regiōes. Pode-se, portanto, concluir que no Brasil a transição epidemiológica para um novo perfil de mortalidade (das doenças infecciosas para as cardiovasculares, neoplasias e causas externas) ocorre com a simultânea persistência, embora quantitativamente diferenciada, de ambos os perfis.

Esta superposição de padrões fica ainda mais evidente, conforme salientou Possas (1989), quando se considera também o perfil de morbidade. As chamadas endemias rurais, por exemplo, quando se urbanizam, incidem e prevalecem desigualmente, atingindo preferencialmente 
as populaçōes mais pobres. Observa-se tambem que, apesar da ocorrida transição epidemiológica, a malária tem alta prevalência em pontos especificos da região Norte (especialmente Rondbnia), a febre amarela comoça a incidir no Centro-Oeste, e o dengue (desde 1987) e o cólera (a partir de 1991) ressurgem no Brasil.

As doenças infecciosas podem, portanto, conforme observou Laurenti (1990), voltar a assumir proporção importante entre as causas de morte. Se assim ocorrer, a tendência histórica observada nas Figuras 1 a 5 pode vir a se modificar nos próximos anos, particularmente onde as desigualdades sociais forem relevantes, uma vez que as doenças infecciosas apresentam maior prevalência nas regiōes de precária infra-estrutura e entre as populaçōes mais pobres.

Alem disso, estudos feitos em paises desenvolvidos, onde a transição epidemiológica se completou há mais tempo, mostraram que mesmo as doenças cardiovasculares, as neoplasias e as causas extemas apresentam incidência e prevalência desiguais entre regiões e grupos populacionais. Esta situação, conforme salientaram Brigs \& Leonard IV (1977), limita a espacidade de reduzir os diferenciais de morbi-mortalidade, algo mais facilmente alcançado através do controle das doenças infecciosas.

$\mathrm{Na}$ Inglaterra, por exemplo, as mortes por causas externas (acidentes), que envolvem predominantemente adultos jovens, foram, segundo Blaxter (1983), sete vezes mais prevalentes entre jovens das classes sociais mais baixas, enquanto as doenças cardiovasculares se mostram mais frequientes entre os operários (Rose \& Marmot, 1981).

No Brasil, similarmente, Costa (1981) mostrou que a hipertensāo arterial tem alta determinaçio social. Estudos desenvolvidos por Costa (1983), no Rio Grande do Sul, sugerem que a proporçāo de trabalbadores de baixa renda em uma população está relacionada com a prevalência da hipertensioo arterial. Também na regiāo metropolitana de Recife, Tandeitnik (1990) observou maior prevalência da hipertensão arterial entre os pacientes da seguridade social (portanto, com menor poder aquisitivo), quando comparados com aqueles da clínica privada.

Enquanto isto, estudos nos paises desenvolvidos (Stallones, 1980) vền demonstrando o declínio da prevalência dos grupos de causas pós-transição epidemiológica (particularmente as cardiovasculares), indicando que uma nova transição estaria se processando naquelas sociedades.

Desta forma, estas doenças não seriam a consequência inevitável do processo de envelhecimento da população e portanto, doenças crônico-degenerativas irredutiveis. Seriam preveníveis por serem o resultado de modifi-

TABELA 1 - Standard Mortality Ratio (SMR) por Grupos de Causas para as Regióes Brasileiras no Ano de 1985

\begin{tabular}{lccccc}
\hline \hline Grupos de Causas & Norte & Nordiste & Centro-Oeste & Sul & Sudeste $^{(9)}$ \\
\hline Infecciosas & 253 & 168 & 159 & 88 & 100 \\
Cardiovasculares & 25 & 56 & 89 & 101 & 100 \\
Neoplasias & 75 & 51 & 87 & 118 & 100 \\
Externas & 103 & 93 & 136 & 96 & 100 \\
\hline \hline
\end{tabular}

(*) Padrăo: Região Sudeste $=100$

Fonte: Prata (1989) 
caçōes, não apenas no estilo de vida, mas tambern da relaçäo do ser humano com o ambiente onde vive e do qual faz parte. Modificaçöes estas que, conforme salientou Doyal (1979), vêm ocorrendo numa velocidade maior do que a capacidade bio-psico-cultural de adaptaçäo is novas circunstâncias.

Esta determinaçäo, relacionada com a ambientação, pode ser ilustrada pela observaçäo de Leal et al. (1985) de que migrantes oriundos de áreas de baixa prevalência de hipertensão arterial, quando vêm a residir $\mathrm{em}$ íreas de alta prevalência, passam a sofiner de hipertensão, com prevalência proporcional ao tempo de migraçäo. Também os estudos de Burkitt (1973), sobre as doenças que se tornaram comuns no mundo desenvolvido a longo deste século, indicam a importância das mudanças culturais e dietéticas ocorridas, particularmente evidentes pelo aumento da incidencia destas mesmas doenças em migrantes de sociedades onde estas eram desconhecidas.

Assim, as mudanças no modelo de desenvolvimento, no estilo de vida e no comportamento assumem importância para a Saúde Pública, estando os pobres, como vimos, em desvantagem em relação aos ricos quanto ao risco concomitante do perfil de morti-mortalidade do atraso (doencas infecciosas) e da modernidade (doenças cardiovasculares e neoplasias). No Brasil, a particularidade da observada transição epidemiológica traz para consideraçäo importantes componentes sócio-econômicos, culturais, demograficos e ambientais.

\section{RESUMO}

PRATA, P. R. A Transição Epidemiolog̣ca no Brasil. Cad. Saúde Públ., Rio de Janeiro, 8 (2): 168-175, abr/jun, 1992.

Tendo como referência a teoria da transiçāo epidemiológica, o autor descreve a evolução da mortalidade no Brasil, avaliando criticamente a modificação do perfil epidemiológico ocortida no Brasil nos últimos 50 anos. O autor revê, situando no contexto brasileiro, a determinação da mortalidade, seja pelo desenvolvimento econômico ou pela introdução de medidas de saúde pública.
Argumenta que as doenças cardiovasculares e as neoplasias estariam tambem relacionadas com fatores ambientais e sócio-culturais, mīo devendo ser consideradas doenças crônico-degenerativas, mas sim preventveis. Estes grupos de causas de morbi-mortalidade tambén sīo considerados com prevaléncin desigual e maior entre as populaçōes mais pobres. $O$ autor faz referencia a uma transiçio epidemiológica que nāo se completou graças d persistência des doenças infeccioses.

Palavras-Chave: Trunsição Epidemiológica; Perfil Epidemiológico; Determinação da Morbi-Mortalidade;

\section{REFERÊNCIAS BIBLIOGRÁFICAS}

ARRIAGA, E. E. \& DAVIS, K., 1969. The pettern of mortality change in Latin America. Demography, 6: 223-242.

BLAXTER, M., 1983. Health services as a defence against poverty in industrialized societies. Social Science and Medicine, 17: 1139-1148.

BRIGGS, R. \& LEONARD IV, W. A., 1977. Mortality and ecological structure: a canonical approach. Social Science and Medicine, 11: 757 762.

BURKITT, D. P., 1973. Some diseases characteristic of modern Western civilization. British Medical Journal, 1: 274-278.

COSTA, E A., 1981. A cross-sectional survey of blood pressure in Rio Grande do Sul, Brazil. Ph.D. Dissertation, Londres: London University.

COSTA, E. A., 1983. Hipertensåo arterial como problema de massa no Brasil: caracteres epidemiológicos e fatores de risco. Ciéncia e Cutura, 35: 1642-1649.

DOYAL, L., 1979. The Political Economy of Health. Londres: Pluto Press.

LAURENTI, R, 1990. Transição demográfica e transição epidemiológica. I Congresso Brasileiro de Epidemiologia, Anais, pp. 143-165, Rio de Janeiro: Abrasco.

LEAL, M. C.; COSTA, E. A.; KLEIN, C. E; SZWARCWALD, C. L. \& BARATA, P. C., 1985. Migração interna e pressão arterial no Rio Grande do Sul. Cadernos de Saúde Pública, 1: 207-219.

McKEOWN, T. \& BROWN, R. G., 1956. Medical evidence related to English population changes in the eighteenth century. Population Studies, 9: 119-141. 
McKEOWN, T. \& RECORD, R. G., 1963. Reasons for the decline of mortality in England and Wales during the nineteenth century. Population Studies, 26: 94-122.

McKEOWN, T.; RECORD, R. G. \& TURNER, R. D., 1975. An interpretation of the decline of mortality in England and Wales during the twentieth century. Population Studies, 24: 391-422.

OMRAM, A. R., 1971. The epidemiologic transition: a theory of the epidemiology of population change. Milbank Memorial Fund Quarterly, 49 (Part 1): 509-538.

PENDLETON, B. F. \& YANG, S., 1985. Socioeconomic and health effects on mortality declines in developing countries. Social Science and Medicine, 20: 453-460.

POSSAS, C., 1989. Epidemiologia e Sociedade, Heterogeneidade e Saúde no Brasil. São Paulo: Hucitec.

PRATA, P. R., 1989. Uneven Development and the Inequality of Mortality in Brazil. Tese de Mestrado, Leeds: Department of Public Health Medicine, Leeds University.

PRESTON, S., 1975. The changing relation between mortality and level of economic development. Population Studies, 29: 231-247.

ROSE, G \& MARMOT, M., 1981. Social class and coronary heart disease. British Heart Journal, 45: 13-19.

STALLONES, R. A., 1980. The rise and fall of ischaemic heart diseases. Scientific American, 243: 43.

TANDEITNIK, L. S., 1990. Epidemiology of Hypertension in Recife, Northeastern Brazil: results from a retrospective study and proposals for preventive measures. Tese de Mestrado, Leeds: Leeds University.

W.H.O. (World Health Organization), 1975. International Classification of Diseases, Injuries and Causes of Death. 9a Revisão, Genebra: WHO.

WOOD, C. H. \& CARVALHO, J. A. M., 1988. The Demography of Inequality in Brazil. Cambridge: Cambridge University Press. 